\title{
Extracranial internal carotid artery dissection caused by compression from a giant osteophyte due to atlantoaxial osteoarthritis: case report
}

\author{
Taichi Ikedo, MD,1,2 Kazuhito Nakamura, MD, ${ }^{1}$ Noritaka Sano, MD, ${ }^{1,2}$ Manabu Nagata, MD,,2 \\ Yumiko Okada, MD, PhD, ${ }^{1}$ Taichiro Kawakami, MD, PhD, ${ }^{3}$ and Takaho Murata, MD, PhD ${ }^{1}$ \\ 1Department of Neurosurgery, Suisyoukai Murata Hospital, Osaka; ${ }^{2}$ Department of Neurosurgery, Kyoto University Graduate \\ School of Medicine, Kyoto; and '3Department of Neurosurgery, Osaka City University Graduate School of Medicine, Osaka, \\ Japan
}

\begin{abstract}
Deformed osseous structures have been reported as rare causes of extracranial internal carotid artery (ICA) dissection, including the styloid process and the hyoid bone. Here, the authors describe the first known case of symptomatic ICA dissection caused by a giant osteophyte due to atlantoaxial osteoarthritis. The left ICA was fixed at the skull base and at the ICA portion compressed by the osteophyte, and it was highly stretched and injured between the two portions during neck rotation. The patient was successfully treated with ligation of the affected ICA following balloon test occlusion. Atlantoaxial osteoarthritis should be considered in the differential diagnosis of ICA dissection in patients with a severely deformed cervical spine.
\end{abstract}

https://thejns.org/doi/abs/10.3171/2017.1.SPINE161047

KEY WORDS internal carotid artery; dissection; atlantoaxial osteoarthritis; osteophyte; cervical

$\mathrm{D}$ ISSECTION of the extracranial internal carotid artery (ICA) is not a common cause of embolic cerebral infarction, accounting for only $0.4 \%-2.5 \%$ of all strokes. ${ }^{15}$ Some atraumatic ICA dissections are the result of rapid or prolonged exertion of the neck. Other cases are attributable to diseases that cause vulnerability of the arterial wall, including hypertension, ${ }^{9}$ fibromuscular dysplasia, ${ }^{2}$ Marfan's syndrome, ${ }^{4}$ Ehlers-Danlos syndrome, ${ }^{4}$ and cystic medial necrosis. ${ }^{18} \mathrm{~A}$ few cases of ICA dissection have been related to repeated compression by deformed osseous structures, such as an elongated styloid process ${ }^{17,21}$ or hyoid bone. ${ }^{22}$

Atlantoaxial osteoarthritis (AAOA) is a degenerative change of osseous structures in the facet joints or central atlantoaxial joints. The prevalence rates of severe AAOA changes in the facet joints have been estimated as $5.4 \%$ in the 6th decade of life and $18.2 \%$ in the 9th decade of life. ${ }^{24}$ Commonly reported clinical symptoms are neck pain, restriction of neck movement, and neck muscle spasm. ${ }^{8}$ Authors of a previous report have described a protruding osteophyte compressing the C-2 nerve root and causing occipital neuralgia. ${ }^{11}$ To our knowledge, however, there have been no reports of compression and dissection of the extracranial ICA by an osteophyte.

Here, we describe a rare case of symptomatic extracranial ICA dissection caused by compression from a giant osteophyte due to AAOA.

\section{Case Report}

History and Examination

A 66-year-old man presented with the chief complaint of dysarthria. Although he had experienced neck pain 10 years earlier, he had only mild neck pain on admission. Physical examination revealed a swelling on the left side of his neck without carotid bruit. Neurological examinations showed mild right paresis as well as ptosis on the side ipsilateral to the swelling, related to Horner's syndrome. He had a history of hypertension and cerebral infarction in the cerebellum but no history of trauma.

ABBREVIATIONS $\mathrm{AAOA}=$ atlantoaxial osteoarthritis; $\mathrm{BTO}=$ balloon test occlusion; $\mathrm{CTA}=\mathrm{CT}$ angiography; $\mathrm{DSA}=$ digital subtraction angiography; $\mathrm{ECA}=$ external carotid artery; ICA = internal carotid artery; MEP = motor evoked potential; MRA = MR angiography; SSEP = somatosensory evoked potential.

SUBMITTED August 30, 2016. ACCEPTED January 5, 2017.

INCLUDE WHEN CITING Published online May 12, 2017; DOI: 10.3171/2017.1.SPINE161047. 

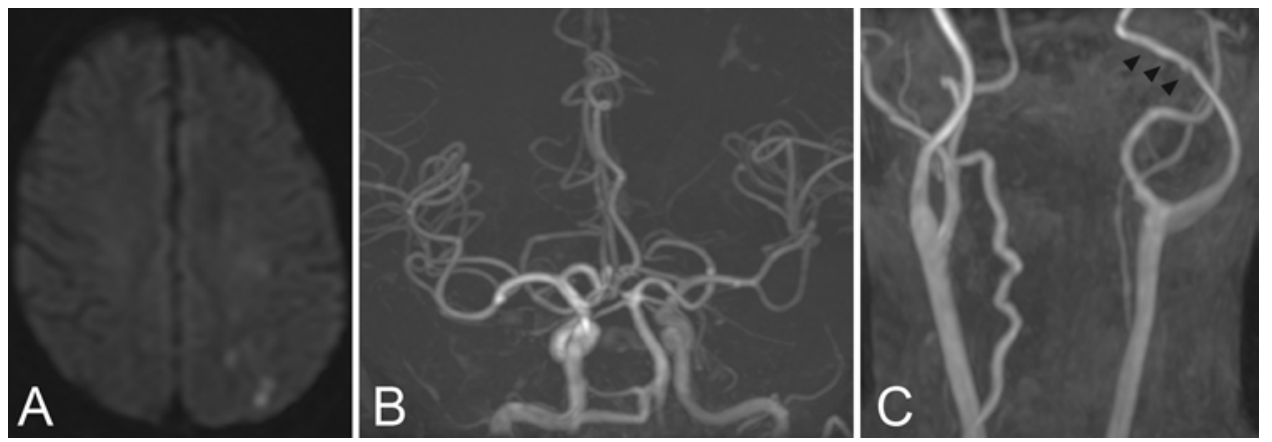

FIG. 1. Admission MRI and MRA studies. A: Multiple fresh cerebral infarcts were detected in the left frontal and parietal lobes on diffusion-weighted imaging. B: The intensity of the left intracranial ICA decreased on intracranial MRA. C: Cervical MRA showed a laterally curved left ICA. Arrowheads indicate the irregularly shaped arterial wall of the left ICA.

\section{Imaging Findings}

Brain MRI and MR angiography (MRA) were performed on admission. On diffusion-weighted images, multiple acute embolic infarcts were detected in the left frontal and parietal lobes (Fig. 1A). The intensity of the left intracranial ICA decreased on intracranial MRA (Fig. 1B). The left cervical ICA was tortuously curved and displaced to the lateral side on MRA. The medial wall of the distal third of the left ICA was irregularly shaped (Fig. 1C). Three-dimensional CT angiography (CTA) revealed a giant osteophyte originating from an enlarged atlantoaxial facet joint (Fig. 2A). The left ICA was highly laterally curved, became stenosed in the laterally displaced portion, and showed a pearl-and-string sign close to the skull base (Fig. 2B). The fusion image of the ICA and the osteophyte indicated that stenosis was caused by compression from the giant osteophyte (Fig. 2C and D). Although 3D CTA detected the dissected ICA with an intramural hematoma close to the skull base, the lesion had no contact with the osteophyte (Fig. 2C and E). Digital subtraction

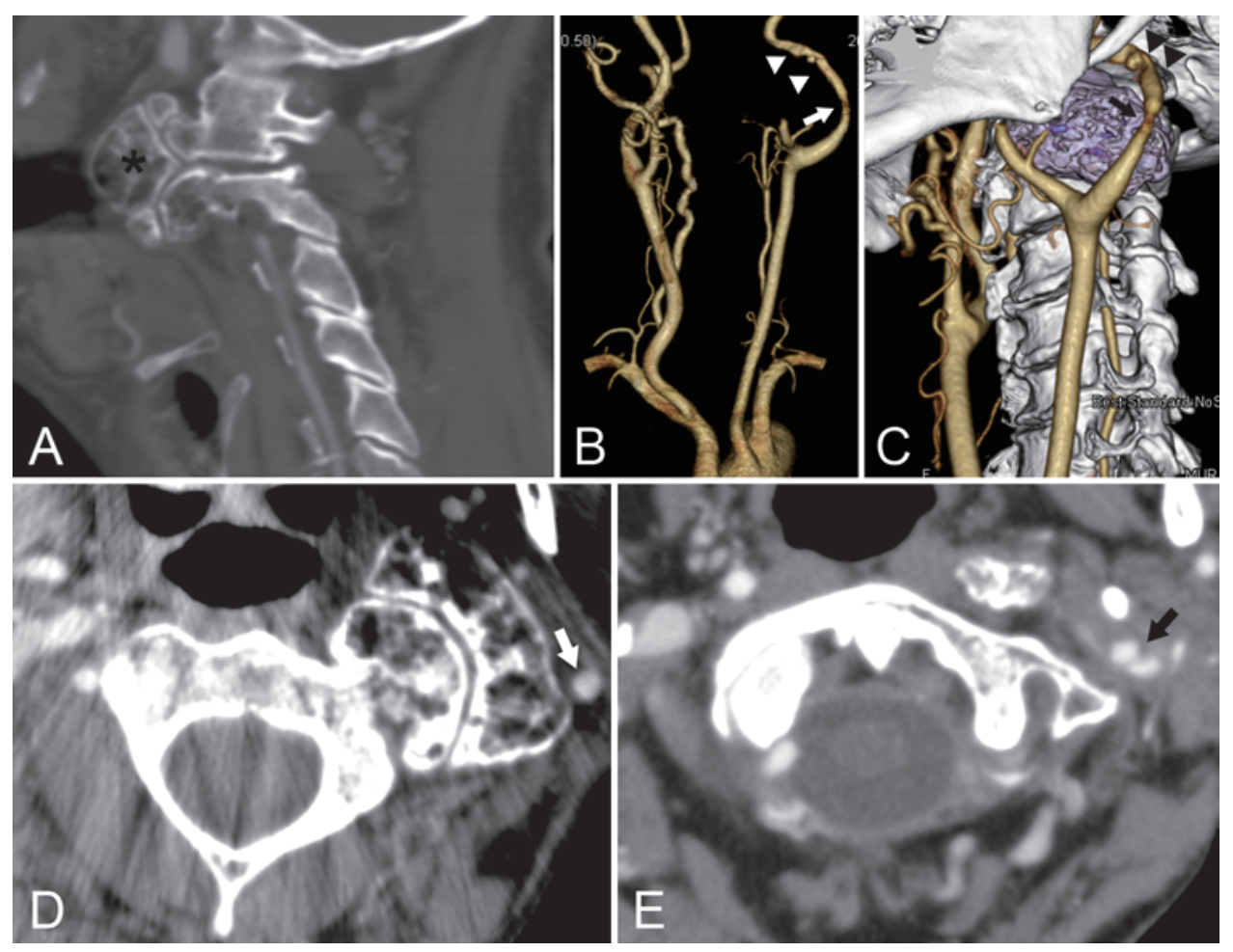

FIG. 2. Admission CT and 3D CTA studies of the cervical lesion. A: Sagittal cervical CT showing a giant osteophyte (asterisk). B: Three-dimensional CTA showing the extracranial ICA. White arrow indicates ICA stenosis. White arrowheads indicate irregularly shaped arterial wall of the left ICA. C: Fusion image of the lesion of the ICA and the osteophyte. The purple mass is the giant osteophyte. Black arrow shows the ICA stenosis due to compression by the osteophyte. Black arrowheads indicate the dissected lesion. D: Axial enhanced cervical CT image showing the ICA (white arrow) laterally compressed by the osteophyte. E: The left ICA was dissected above the mass lesion. Black arrow indicates an enhanced pseudolumen. Figure is available in color online only. 


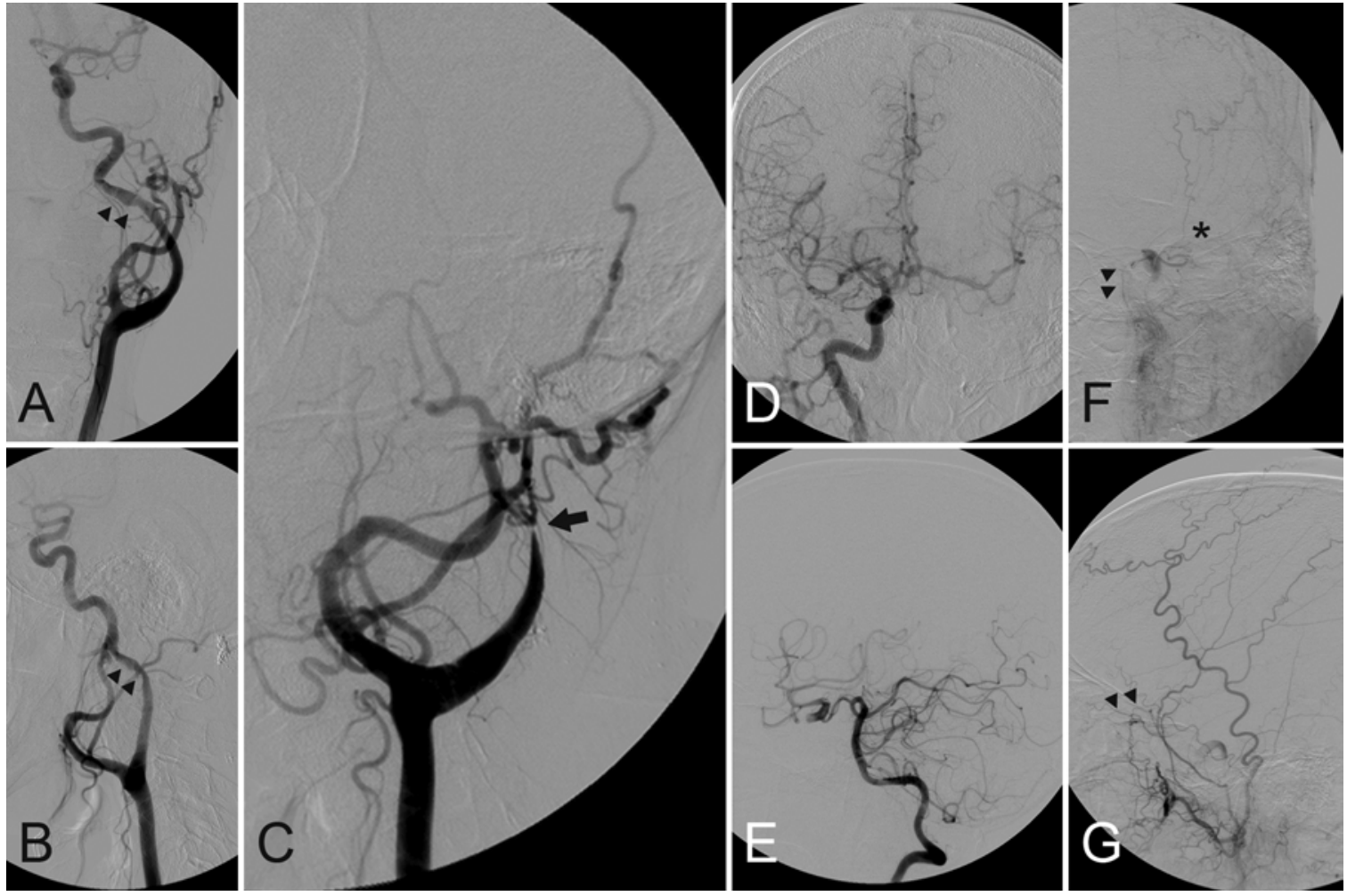

FIG. 3. Frontal (A) and lateral (B) DSA images of the left ICA, with arrowheads indicating the dissected point of the ICA. Neck rotation to the left occluded the left ICA (black arrow, C). Results of the BTO indicated that the right ICA supplied the left cerebrum via the anterior communicating artery (D); the left cerebrum was supplied via the posterior communicating artery (lateral image of right vertebral artery, E); and the internal maxillary artery supplied the left ICA via the ethmoidal artery (arrowheads, frontal [F] and lateral [G] views of left superficial temporal artery). The superficial temporal artery supplied the ICA via the superior orbital artery (asterisk, F).

angiography (DSA) was performed on the 12th day after admission, following antiplatelet medication for acute cerebral infarction. Digital subtraction angiography showed the dissected ICA just proximal to the foramen lacerum (Fig. 3A and B). Neck rotation to the left occluded the compressed portion of the left ICA (Fig. 3C), although the occlusion was reversible on contralateral rotation.

The patient was diagnosed with cerebral infarction due to temporary occlusion and dissection of the left extracranial ICA caused by AAOA. Antiplatelet therapy was continued and rotation of the neck was restricted using a neck brace. Surgical treatment for the embolic source was planned to prevent the recurrence of cerebral infarction.

\section{Balloon Test Occlusion}

Balloon test occlusion (BTO) of the left extracranial ICA was performed to examine tolerance for ischemia (Fig. 3D-G). Right common carotid artery angiography and right vertebral artery angiography during BTO indicated that the left intracranial ICA was sufficiently supplied through the anterior and posterior communicating artery, respectively (Fig. 3D and E). The frontal branch of the left superficial temporal artery supplied the left intracranial ICA via the superior orbital artery. The left internal maxillary artery supplied the left intracranial ICA via the ethmoidal artery (Fig. 3F and G). Xenon CT did not show decreased blood flow in the left hemisphere during BTO of the left ICA.

\section{Operative Procedure}

We planned to ligate the left ICA without external carotid artery (ECA)-ICA anastomosis based on the results of BTO. The cervical ICA was exposed with the patient under general anesthesia. Somatosensory evoked potentials (SSEPs) and transcranial motor evoked potentials (MEPs) were used for monitoring test occlusion for 20 minutes. The SSEPs were checked every 2 minutes and the MEPs every 10 minutes. No remarkable changes in SSEPs or MEPs were detected during occlusion of the left ICA using bulldog forceps. Finally, we performed ligation of the left ICA with 1-0 silk sutures without ECA-ICA anastomosis.

\section{Postoperative Course}

The patient recovered from anesthesia 66 minutes after ligation of the left ICA and had no new neurological deficits or impaired swallowing after surgery. Postoperative MRI showed no fresh hemorrhage or infarct (Fig. 4A). Ligation of the left ICA was confirmed on MRA images (Fig. 4B and C). Over the subsequent 3 years, there have been no signs of recurrent stroke.

\section{Discussion}

Here, we reported a rare case of ICA dissection related to repeated compression from a giant osteophyte on the facet joint of C-1 and C-2. The patient was diagnosed with 

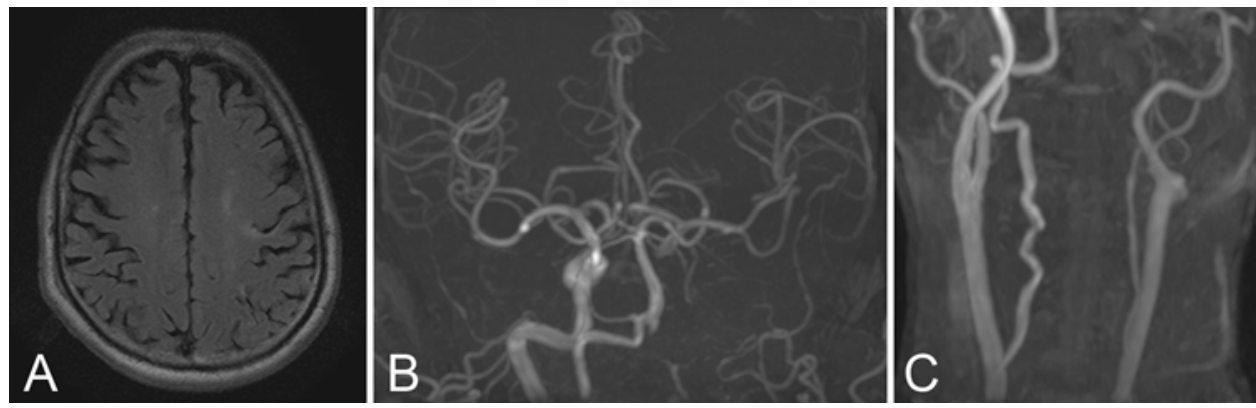

FIG. 4. Postoperative MRI and MRA studies. A: Axial FLAIR MR image showing no fresh hemorrhage or infarct. B: Intracranial MRA confirming ligation of the left ICA. C: Cervical MRA showing the ligated left ICA.

AAOA using imaging studies, including 3D CTA, MRI, and DSA. We verified sufficient collateral flow from the contralateral ICA, the posterior circulation, and the ECA during test occlusion of the ICA. The patient was successfully treated with ligation of the ICA and antiplatelet medication without removal of the giant osteophyte. To our knowledge, this is the first reported case of successfully treated symptomatic ICA dissection caused by a giant osteophyte due to AAOA.

Painful AAOA is initially treated with nonsurgical procedures, such as physical therapy, immobilization, medication, and anesthetic injection. ${ }^{14}$ Medications including nonsteroidal antiinflammatory drugs, steroids, or gabapentin ${ }^{1}$ have been reported to be effective. Patients with intractable pain despite nonsurgical therapies have been treated surgically; for example, posterior atlantoaxial fusion via a single surgery was successfully performed in 98\% of cases, and preoperative neck pain improved in $97.7 \%$ of the patients. ${ }^{6}$ As fusion surgery is effective for intractable pain, the irritation within the degenerated joint has been assumed to be one of the main causes of pain.? In the present case, the patient had neck pain 10 years earlier with progression of AAOA. We believed that the natural fusion of the facet joint by the enlarged osteophyte decreased the irritation within the degenerated joint and his intractable neck pain. This unusual course may have contributed to the formation of the rare giant osteophyte, which was not noticed until the development of multiple infarcts from the ICA dissection.

The pathologies caused by the lesion in the present case were stenosis, temporary occlusion, and dissection of the left ICA due to the giant osteophyte. The stenosis and occlusion were related to direct compression by the osteophyte, with neck rotation in common with the vertebral artery occlusion frequently seen in bow hunter's syndrome. ${ }^{23}$ With regard to the mechanisms of spontaneous arterial dissection, ICA dissection has been reported to result from injury to the intima or bleeding from the vasa vasorum..$^{20}$ Curiously, in a previous report, ${ }^{3}$ the length of the ICA increased and its diameter decreased with maximum head rotation because the ICA was fixed at the carotid bifurcation and the osseous carotid canal at the skull base. Wall stress of the intimal side of the ICA increased at the skull base and the cranial edge of the carotid sinus. Downer et al..$^{5}$ reported that $36(94.7 \%)$ of 38 vessels had maximum mural hematoma thickness in the distal third of the ICA and that fixation of the ICA at the cranial base was related to the pathology of ICA dissection. In the present case, the left ICA was fixed at the skull base and at the ICA portion compressed by the osteophyte and was highly stretched between these two portions during neck rotation. These phenomena suggest that wall stress on the distal extracranial ICA caused the dissection.

A previous report indicated that $58 \%$ of 172 consecutive patients with ICA dissection suffered acute cerebral infarction and that $85 \%$ of cases with fresh infarcts had embolic stroke. ${ }^{13}$ As occlusion of the left ICA was temporary during neck rotation and collateral flow was sufficient, our patient was diagnosed with cerebral infarction caused by thrombus formation in the affected ICA. Although treatment for ICA dissection with antiplatelets versus anticoagulants has been a matter of debate, a novel randomized trial, the Cervical Artery Dissection in Stroke Study, concluded that there was no difference between the two medications in preventing stroke or death. ${ }^{10,12}$ In the present case, antiplatelet therapy was selected for the prevention of recurrence to avoid adverse events, including intracranial hemorrhage or extension of the intramural hemorrhage. As most cases are benign and rates of recurrent dissection and stroke are estimated to be about $1.1 \%$ and $8.5 \%$, respectively, ${ }^{16}$ nonsurgical therapies are initially considered for ICA dissection. However, we decided to treat our patient surgically because repeated arterial compression is related to a high risk of arterial injury and clot formation. ${ }^{19}$

To alleviate repeated compression of the $\mathrm{C}-2$ nerve root, a surgical approach to the osteophyte and atlantoaxial fusion was performed in another case. ${ }^{11}$ However, for compression of the ICA, we believed that $\mathrm{C} 1-2$ fusion would not be enough to prevent repeated arterial injury and occlusion by neck rotation. Notably, in the present case, we planned to perform less invasive surgery to avoid the risk of drilling the osteophyte close to the displaced ICA. As the tortuously curved lesion with vulnerable thrombus was not suitable for stent placement via an endovascular approach, we chose trapping surgery. Although endovascular trapping with coil embolization would have also been a viable option, we planned ligation with direct surgery so that we would be able to discontinue trapping after the start of ligation even if the patient showed unexpected neurological symptoms. We performed BTO and cerebral blood flow study and confirmed tolerance for ischemia and collateral flow. Intraoperative MEPs and SSEPs assisted in safe ligation of the left ICA without ECA-ICA 
anastomosis. The disadvantage of our strategy was an inability to monitor verbal function during the operation. We believe that the operation should ideally be performed while monitoring verbal function or with the patient under local anesthesia.

Our strategy was successful in treating a patient with this rare pathology. Atlantoaxial osteoarthritis should be considered in the differential diagnosis of ICA dissection in patients with a severely degenerated and deformed cervical spine.

\section{References}

1. Ak H, Özlem B, Aylin O: Effectiveness of gabapentin in unilateral atlantoaxial osteoarthritis as a cause of painful neck. World Neurosurg 81:e16-e18, 2014

2. Assadian A, Senekowitsch C, Assadian O, Schuster H, Ptakovsky H, Hagmüller GW: Combined open and endovascular stent grafting of internal carotid artery fibromuscular dysplasia: long term results. Eur J Vasc Endovasc Surg 29:345-349, 2005

3. Callaghan FM, Luechinger R, Kurtcuoglu V, Sarikaya H, Poulikakos D, Baumgartner RW: Wall stress of the cervical carotid artery in patients with carotid dissection: a case-control study. Am J Physiol Heart Circ Physiol 300:H1451H1458, 2011

4. Debette S, Goeggel Simonetti B, Schilling S, Martin JJ, Kloss M, Sarikaya H, et al: Familial occurrence and heritable connective tissue disorders in cervical artery dissection. Neurology 83:2023-2031, 2014

5. Downer J, Nadarajah M, Briggs E, Wrigley P, McAuliffe W: The location of origin of spontaneous extracranial internal carotid artery dissection is adjacent to the skull base. J Med Imaging Radiat Oncol 58:408-414, 2014

6. Elliott RE, Tanweer O, Smith ML, Frempong-Boadu A: Outcomes of fusion for lateral atlantoaxial osteoarthritis: meta-analysis and review of literature. World Neurosurg 80:e337-e346, 2013

7. Finn M, Fassett DR, Apfelbaum RI: Surgical treatment of nonrheumatoid atlantoaxial degenerative arthritis producing pain and myelopathy. Spine (Phila Pa 1976) 32:3067-3073, 2007

8. Goel A, Shah A, Gupta SR: Craniovertebral instability due to degenerative osteoarthritis of the atlantoaxial joints: analysis of the management of 108 cases. J Neurosurg Spine 12:592-601, 2010

9. Gonzales-Portillo F, Bruno A, Biller J: Outcome of extracranial cervicocephalic arterial dissections: a follow-up study. Neurol Res 24:395-398, 2002

10. Gross BA, Albuquerque FC: Antiplatelets versus anticoagulation for cervical arterial dissection. World Neurosurg 84:21-22, 2015

11. Guha D, Mohanty C, Tator CH, Shamji MF: Occipital neuralgia secondary to unilateral atlantoaxial osteoarthritis: Case report and review of the literature. Surg Neurol Int 6:186, 2015

12. Markus HS, Hayter E, Levi C, Feldman A, Venables G, Norris J: Antiplatelet treatment compared with anticoagulation treatment for cervical artery dissection (CADISS): a randomised trial. Lancet Neurol 14:361-367, 2015 (Erratum in Lancet Neurol 14:566, 2015)
13. Morel A, Naggara O, Touzé E, Raymond J, Mas JL, Meder $\mathrm{JF}$, et al: Mechanism of ischemic infarct in spontaneous cervical artery dissection. Stroke 43:1354-1361, 2012

14. Ogoke BA: The management of the atlanto-occipital and atlanto-axial joint pain. Pain Physician 3:289-293, 2000

15. Provenzale JM: Dissection of the internal carotid and vertebral arteries: imaging features. AJR Am J Roentgenol 165:1099-1104, 1995

16. Schwartz NE, Vertinsky AT, Hirsch KG, Albers GW: Clinical and radiographic natural history of cervical artery dissections. J Stroke Cerebrovasc Dis 18:416-423, 2009

17. Sveinsson O, Kostulas N, Herrman L: Internal carotid dissection caused by an elongated styloid process (Eagle syndrome). BMJ Case Rep 2013:bcr009878, 2013

18. Thal DR, Schober R, Schlote W: Carotid artery dissection in a young adult: cystic medial necrosis associated with an increased elastase content. Clin Neuropathol 16:180-184, 1997

19. Thomas B, Barreau X, Pointillart V, Sibon I, Renou P: Endovascular embolization of a nondominant vertebral artery compressed by an osteophyte to prevent recurrence of vertebrobasilar infarctions. J Stroke Cerebrovasc Dis 24:e257e259, 2015

20. Völker W, Dittrich R, Grewe S, Nassenstein I, Csiba L, Herczeg L, et al: The outer arterial wall layers are primarily affected in spontaneous cervical artery dissection. Neurology 76:1463-1471, 2011

21. Yamamoto S, Todo K, Kawamoto M, Kohara N: Carotid artery dissection associated with an elongated styloid process. Intern Med 52:1005-1006, 2013

22. Yukawa S, Yamamoto S, Hara H: Carotid artery dissection associated with an elongated hyoid bone. J Stroke Cerebrovasc Dis 23:e411-e412, 2014

23. Zaidi HA, Albuquerque FC, Chowdhry SA, Zabramski JM, Ducruet AF, Spetzler RF: Diagnosis and management of bow hunter's syndrome: 15-year experience at Barrow Neurological Institute. World Neurosurg 82:733-738, 2014

24. Zapletal J, de Valois JC: Radiologic prevalence of advanced lateral C1-C2 osteoarthritis. Spine (Phila Pa 1976) 22:2511-2513, 1997

\section{Disclosures}

The authors report no conflict of interest concerning the materials or methods used in this study or the findings specified in this paper.

\section{Author Contributions}

Conception and design: Nakamura. Acquisition of data: Nakamura, Kawakami. Analysis and interpretation of data: Ikedo. Drafting the article: Ikedo. Critically revising the article: Ikedo, Nakamura. Reviewed submitted version of manuscript: Ikedo, Sano, Nagata. Approved the final version of the manuscript on behalf of all authors: Ikedo. Study supervision: Nakamura, Sano, Nagata, Okada, Kawakami, Murata.

\section{Correspondence}

Taichi Ikedo, Department of Neurosurgery, Suisyoukai Murata Hospital, 4-2-1, Tajima, Ikuno-ku, Osaka 544-0011, Japan. email: taichi.ikedo@gmail.com. 